\title{
Estudo da mistura do éter diglicidílico do bisfenol-A com poli(etileno-co-acetato de vinila)-g-poli(metacrilato de metila) por espectroscopia dielétrica e calorimetria exploratória diferencial
}

\author{
Study of the blend of diglycidyl ether of bisphenol-A with copolymer of \\ poly(ethylene-co-vinyl acetate)-g-poly(methyl methacrylate) by dielectric \\ spectroscopy and differential scanning calorimeter
}

Leyva, M.E. ${ }^{1}$; Garcia, F.G. ${ }^{1}$; Soares, B.G. ${ }^{2}$

\author{
${ }^{1}$ Laboratório de Biomateriais, Departamento de Química e Física, Instituto de Ciências Exatas, \\ Universidade Federal de Itajubá. Av. BPS, nº. 1303, Pinheirinho CEP: 37500-903, Itajubá, M.G., Brasil. \\ e-mail: mariae@unifei.edu.br ; fili@unifei.edu.br \\ ${ }^{2}$ Instituto de Macromoléculas, Universidade Federal do Rio de Janeiro, Centro de Tecnologia, \\ B1. J, Ilha do Fundão, 21945-970, Rio de Janeiro, R.J., Brasil. \\ e-mail: bluma@ima.com.br
}

\begin{abstract}
RESUMO
As curvas calorimétricas e os espectros de relaxação dielétrica correspondentes às misturas do éter diglicidílico do bisfenol-A com diferentes concentrações do copolímero poli(etileno-co-acetato de vinila)-gpoli(metacrilato de metila) (EVA-g-PMMA) foram obtidos utilizando a calorimetria exploratória diferencial (DSC), e a espectroscopia de relaxação dielétrica (DSR), respectivamente. A temperatura de transição vítrea $\left(T_{\mathrm{g}}\right)$ do segmento de PMMA aumenta com o conteúdo de copolímero na mistura. O comportamento da $T_{\mathrm{g}}$ foi descrito pela equação de Gordon-Taylor, utilizando a fração mássica de PMMA. Nos espectros dielétricos, aparecem as relaxações do tipo $\alpha$ características dos dois segmentos moleculares. Neste caso, o aumento da concentração de copolímero provocou o aumento da temperatura de relaxação $\alpha$ correspondente ao segmento de PMMA, e não foi observada mudança na relaxação $\alpha$ para a fração de EVA. Estes comportamentos revelam que os segmentos estruturais do copolímero apresentam interações bem diferenciadas com o monômetro epoxídico. O segmento de PMMA é miscível, portanto, provocou fortes interações com a resina quando comparado à fração de EVA, que não manifestou interação aparente e originou a heterogeneidade do sistema.
\end{abstract}

Palavras-chave: Miscibilidade, éter diglicidílico do bisfenol-A, copolímero graftizado, calorimetria exploratória diferencial, espectroscopia dielétrica.

\footnotetext{
ABSTRACT

The calorimetric curves and the dielectric relaxation spectra corresponding to blends of diglycidyl ether of bisfenol-A with different concentration of poly(ethylene-co-vinyl acetate)-g-poly(methyl methacrylate) (EVA-g-PMMA) were obtained using differential scanning calorimetry (DSC) and dielectric relaxation espectroscopy (DSR), respectively. The glass transition temperature $\left(T_{\mathrm{g}}\right)$ of the PMMA segment of increases with the copolymer content in the blends. The $T_{\mathrm{g}}$ behavior was described by the Gordon-Taylor equation, using the weight fraction of PMMA. In the dielectric relaxation spectrum, $\alpha$ relaxations of the two molecular segments are deserved. In this case, the increase in copolymer concentration led to the increase of $\alpha$ relaxation corresponding to the PMMA segment, and there was not change in $\alpha$-relaxation for the EVA fraction. These behaviors show that the structural segments have very different interactions with the epoxy monomer. The PMMA segment of is miscible in the epoxy resin, therefore, provoked strong interactions with the resin when compared to EVA fraction, which presented no apparent interaction and gave origin to the system heterogenity.
} 
Keywords: Miscibility, diglycidyl ether of bisphenol-A, grafting copolymer, differential scanning calorimetry, dielectric spectrum.

\section{INTRODUÇÃO}

A utilização de diferentes tipos de modificadores tais como; elastômeros funcionalizados, termoplásticos e copolímeros com diferente arquitetura macromolecular em bloco e/ou graftizados é de grande interesse para o melhoramento da alta rigidez e baixa resistência à fratura de polímeros epoxídicos. Neste sentido os modificadores dispersos na matriz melhoram as propriedades tais como, tenacidade à fratura, flexibilidade, resistência à tração e resistência adesiva [1-5].

Para o melhoramento das propriedades mecânicas de polímeros epoxídicos é usual a utilização de modificadores os que originam uma segunda fase dispersa na matriz. Isto pode ser realizado por dois métodos clássicos. No primeiro, o modificador é solúvel no sistema epoxídico no início da reação. Neste caso, a dispersão e/ou formação da segunda fase do modificador, ocorre por separação de fases induzida por reação química, ou seja, o modificador precipita durante o processo de cura a uma conversão particular. Neste caso, as propriedades podem ser controladas pela solubilidade do modificador no sistema, a concentração do modificador, as condições de cura, etc. Estes fatores afetam o tamanho e distribuição de tamanhos das partículas dispersas (morfologia). No segundo, são utilizadas partículas do modificador insolúveis, as que são previamente formadas (core-shell) e, dispersas inicialmente no sistema. Portanto, neste caso tem se uma dispersão de partículas do modificador desde o início com tamanho e dispersão de tamanhos conhecida [6]. Existe outra possibilidade, que foi relatada pela primeira vez por nosso grupo de pesquisa que consiste na utilização de um copolímero (EVA-g-PMMA) (obtido via radical livre utilizando agente de transferência de cadeia para introduzir os segmentos de PMMA) que apresenta segmentos com diferente solubilidade, neste caso desde o início existe uma ligeira turvação no sistema $(\leq 15 \%$ em massa de copolímero) que desaparece quando o sistema é aquecido e, neste caso a dispersão do modificador na matriz ocorre por separação de fases induzida por reação química [7, 8]. Neste caso particular, foi comprovado que é possível gerar partículas bifásicas estáveis na matriz epoxídica e, que a fração solúvel separa de fases durante a reação química.

Em nosso laboratório foi realizada pela primeira vez a síntese de um copolímero graftizado de poli(etileno-co-acetato de vinila)-g-poli(metacrilato de metila) (EVA-g-PMMA) com segmentos de poli(metacrilato de metila) de tamanho controlado, utilizando a polimerização controlada via radical livre por transferência atômica (ATRP) como uma rota para a síntese de copolímeros com estruturas de massa molecular desejada [9]. A utilização de modificadores para polímeros epoxídicos usando o método de separação de fases induzida por reação química deve ser acompanhada por uma série de estudos preliminares, visando estabelecer a relação entre a morfologia e, por exemplo, as propriedades adesivas [3,10].

Para o controle da morfologia gerada pelo método de separação de fases induzida por reação química é necessário conhecer o diagrama de transformação conversão $(\mathrm{X})$ vs composição do modificador $\left(\phi_{\mathrm{M}}\right)$ a temperatura constante, o qual descreve este processo de modo quantitativo [6]. Entretanto, pode ser obtida informação relativa à possível interação do modificador com os monômeros por diferentes técnicas analíticas. Entre as técnicas utilizadas para obter informação das interações entre os componentes em misturas poliméricas estão: a calorimetria exploratória diferencial (DSC), a espectroscopia de relaxação dielétrica (DRS), entre outras. Para o limite de nosso conhecimento as potencialidades da combinação destas duas técnicas analíticas para avaliar as interações em misturas poliméricas não são claramente divulgadas na literatura científica. Este foi o motivo de nos propor avaliar o grau de interação dos segmentos do copolímero (EVA-g-PMMA) obtido por polimerização controlada via radial livre (ATRP) com o monômero epoxídico do tipo éter diglicidílico do bisfenol A usando estas duas técnicas.

\section{MATERIAIS E MÉTODOS}

A resina utilizada foi o éter diglicidílico do bisfenol A, (DGEBA, DER 331) com $191 \mathrm{~g} \mathrm{eq}^{-1}$., determinado por titulação química e por uma metodologia divulgada por nosso grupo que utiliza a espectroscopia de ressonância magnética nuclear de hidrogênio [11]. A resina foi desidratada por tratamento a vácuo a $80{ }^{\circ} \mathrm{C}$ até o desaparecimento das bolhas. O copolímero de EVA-g-PMMA foi obtido por polimerização controlada via radical livre por transferência atômica (ATRP) [8]. O conteúdo de poli(acetato de vinila) (PVAc) no copolímero EVA (poli(etileno-co-acetato de vinila) é de 40,5\% (em massa), o conteúdo de PMMA no 
copolímero graftizado é de 60,5 \% (em massa) e a massa molecular do segmento graftizado de PMMA é Mn $=74400$ com $\mathrm{MWD}=1,2$.

Misturas binárias com diferentes conteúdos de copolímero foram preparadas a partir de soluções de cada componente em diclorometano. O solvente foi evaporado cuidadosamente a vácuo a $80{ }^{\circ} \mathrm{C}$ até obter um valor de massa constante. Para a obtenção dos espectros dielétricos, foram preparados filmes de $25 \mathrm{~mm}$ x 25 mm x $1 \mathrm{~mm}$ utilizando moldagem por compressão a $160^{\circ} \mathrm{C}$ e 5 Ton durante 10 minutos.

A temperatura de transição vítrea $\left(T_{g}\right)$ das misturas foi determinada utilizando o analisador térmico Perkin-Elmer DSC-7 em atmosfera de nitrogênio a $10{ }^{\circ} \mathrm{C} \min ^{-1}$, na faixa de -27 até $150{ }^{\circ} \mathrm{C}$. A temperatura de transição vítrea $(T \mathrm{~g})$ foi considerada no ponto médio onde é observada a transição na curva calorimétrica obtida na segunda varredura dinâmica. Os espectros de relaxação dielétrica foram obtidos utilizando o analisador termo-dielétrico, TA Instruments 2970, empregando eletrodos de placas paralelas em atmosfera de nitrogênio. A perda dielétrica ( $\left.\varepsilon^{\prime \prime}\right)$ foi obtida no modo dinâmico a $3{ }^{\circ} \mathrm{C} \min ^{-1}$ na faixa -130 a $200{ }^{\circ} \mathrm{C}$ a $10^{5}$ Hz.

\section{RESULTADOS}

\subsection{Calorimetria exploratória diferencial (DSC)}

A Figura 1 apresenta o comportamento da $T_{g}$ das misturas de resina epoxídica do tipo éter diglicidílico do bisfenol-A (DGEBA) com o copolímero EVA-g-PMMA (fração mássica de PMMA) utilizando a equação de Gordon-Taylor.

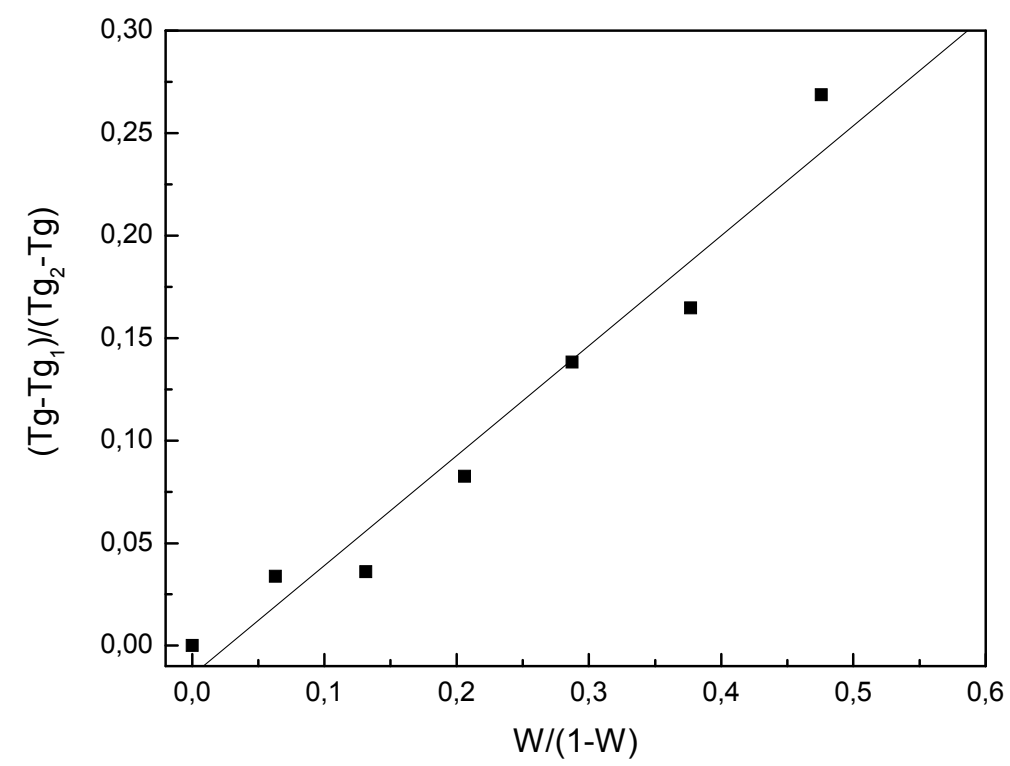

Figura 1: Comportamento da $T_{g}$ das misturas de resina epoxídica com o copolímero de EVA-g-PMMA utilizando a equação de Gordon-Taylor (considerando a fração mássica de PMMA).

\subsection{Espectroscopia de relaxação dielétrica (DRS)}

A Figura 2 mostra de modo comparativo, os espectros de relaxação dielétrica do copolímero, e de três misturas contendo diferentes concentrações de copolímero (\% em massa). 


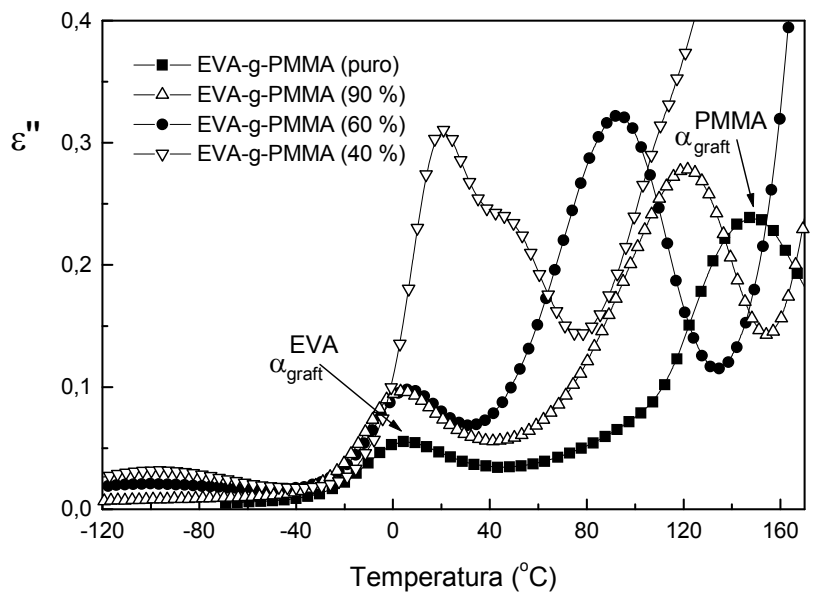

Figura 2: Espectros de relaxação dielétrica do copolímero EVA-g-PMMA e de três misturas de monômero epoxídico contendo $40 \%, 60 \%$ e $90 \%$ (em massa) de copolímero como mostrado na figura.

\section{DISCUSSÃO}

\subsection{Calorimetria exploratória diferencial (DSC)}

Uma característica comum para todas as misturas preparadas foi que a temperatura ambiente $\left(25^{\circ} \mathrm{C}\right)$ existe uma ligeira turvação, o que afetou a transparência das misturas. Entretanto, esta característica foi menos significativa para as misturas contendo concentrações de copolímero baixas ( $\leq 15 \%$, em massa), que quando aquecidas ficaram transparentes. Esta faixa de concentrações é a utilizada para modificar polímeros epoxídicos, pois quando são utilizados termoplásticos como modificadores estes manifestam uma viscosidade elevada. A turvação das misturas era esperada, pois o copolímero graftizado apresenta uma fração (EVA, $40,5 \%$ em massa de PVAc) que não é solúvel na resina, o que significa que não existe total solubilidade do copolímero no monômero. Entretanto, para copolímeros de EVA contendo 70 \% (em massa) de PVAc aparece divulgado que são solúveis na resina do tipo éter diglicidílico do bisfenol A (DGEBA) [12].

Nas condições das análises, não foi possível observar nas curvas calorimétricas a temperatura de transição vítrea $\left(T_{g}\right)$ relativa à fração de EVA do copolímero. Isto impossibilitou obter informação por esta técnica experimental, das interações da fração de EVA com a resina. No entanto, a $T_{g}$ correspondente ao segmento graftizado de PMMA no copolímero foi observada em todas as curvas. A $T_{g}$ aumenta com o aumento da concentração do copolímero. Este comportamento da $T_{g}$ é típico de misturas miscíveis. A equação de Gordon-Taylor (equação 1), pode ser utilizada para descrever o comportamento experimental da $T_{g}$ em misturas poliméricas quando os componentes são miscíveis. Segundo a literatura o PMMA é solúvel na resina do tipo éter diglicidílico do bisfenol-A (DGEBA) [8,13-15]. Para misturas binárias de DGEBA e PMMA tem sido relatado que o valor de $\mathrm{k}$ é 0,20 [13], 0,26 [8,14] e 0,29 [15], onde os valores baixos indicam fortes interações entre os componentes.

$$
\left(\operatorname{Tg}-\operatorname{Tg}_{1}\right) /\left(\operatorname{Tg}_{2}-\mathrm{Tg}\right)=\mathrm{kw} /(1-\mathrm{w})
$$

Onde $\mathrm{Tg}_{1}$ e $\mathrm{Tg}_{2}$ correspondem à $T_{g}$ da resina epoxídica $(253 \mathrm{~K})$ e à $T_{g}$ do segmento de PMMA puro $(388 \mathrm{~K})$, e w corresponde à fração mássica do PMMA na mistura.

A equação 1 foi utilizada para descrever o comportamento do segmento de PMMA no copolímero na mistura com a resina epoxídica. Para isto, foi conveniente utilizar a fração mássica de PMMA nas misturas. Isto foi determinado a partir do conteúdo de PMMA no copolímero (60,5 \% em massa). A Figura 1 mostra este resultado. Uma boa correlação linear $\left(\mathrm{R}^{2}=0,9975\right)$ foi obtida para $\mathrm{k}=0,53$. Este valor relativamente alto indica que a interação do segmento de PMMA do copolímero é menor quando comparado ao PMMA puro. Este comportamento está relacionado à fração de EVA (cadeia principal), que afeta a solubilidade do copolímero e, portanto diminui a interação do segmento de PMMA com o monômero epoxídico. Este valor está de acordo com o encontrado anteriormente $(\mathrm{k}=0,56)$ para uma mistura de DGEBA com um copolímero 
de EVA-g-PMMA neste caso, obtido via radical livre usando agente de transferência para introduzir os segmentos de PMMA na cadeia do copolímero EVA [7].

\subsection{Espectroscopia de relaxação dielétrica (DRS)}

A Figura 2 mostra de modo comparativo os espectros de relaxação dielétrica do copolímero e de três misturas contendo diferentes concentrações de copolímero. Os espectros são representados em termos da perda dielétrica $(\varepsilon ")$ vs temperatura $(\mathrm{T})$, para uma frequência de $10^{5} \mathrm{~Hz}$. Estes espectros fornecem informação da solubilidade dos segmentos do copolímero na resina. Nos espectros dielétricos das misturas foram observadas as relaxações correspondentes tanto da fração de EVA (cadeia principal) quanto da fração relativa ao segmento de PMMA (enxerto). Estes resultados demonstram que esta técnica é mais efetiva para este sistema quando comparada à calorimetria exploratória diferencial para avaliar as interações dos segmentos do copolímero com a resina epoxídica (DGEBA). Oportuno esclarecer que esta técnica resulta mais conveniente para o estudo de misturas poliméricas quando os componentes apresentam atividade dielétrica. Esta atividade está diretamente relacionada à estrutura química e polaridade, que podem controlar a resposta dielétrica em polímeros [16].

No espectro do copolímero puro mostrado na Figura 2, podem ser observadas duas relaxações: a primeira aparece na faixa de $-27 \mathrm{a}-3{ }^{\circ} \mathrm{C}$ atribuída à $T_{g}$ (relaxação $\alpha$ ) da fração de EVA; e a segunda ao redor de $148{ }^{\circ} \mathrm{C}$, correspondente à $T_{g}$ (relaxação $\alpha$ ) do segmento de PMMA. Pode ser observado ainda que a intensidade relativa da relaxação correspondente à fração de EVA é baixa $(\varepsilon " \sim 0,05)$. Isto é consequência da natureza estrutural deste segmento constituído por um copolímero aleatório, com duas unidades diferentes em polaridade, poli(etileno) (apolar) e poli(acetato de vinila) (polar). Neste caso, a fração de poli(acetato de vinila) é a responsável pelo sinal dielétrico da fração EVA [17].

Na Figura 2 pode ser observado também que, para as misturas com $60 \%$ e $90 \%$ (em massa) de copolímero, o valor da $T_{g}$ (relaxação $\alpha$ ) da fração de EVA não muda quando comparada à do copolímero puro. Este resultado indica que para altas concentrações de copolímero, a fração de EVA não apresenta interações aparentes com a resina epoxídica, o que justifica que seja insolúvel. Por outro lado, o valor da $T_{g}$ (relaxação $\alpha$ ) correspondente ao segmento de PMMA aumenta com o aumento da concentração do copolímero. Este comportamento era esperado e coincide com os resultados obtidos por calorimetria exploratória diferencial, onde esta fração é solúvel e apresenta fortes interações com o monômero epoxídico. Estes resultados estão de acordo com o divulgado na literatura, de que para uma mistura miscível a mudança da concentração de polímero deve deslocar o valor da $T_{g}$ (relaxação $\alpha$ ) das misturas [18].

Para o caso particular da mistura contendo $40 \%$ (em massa) de copolímero (Figura 2), a $T_{g}$ relativa ao segmento de PMMA aparece na faixa de -27 a $75{ }^{\circ} \mathrm{C}$ e, apresenta-se na forma de uma curva bimodal com intensidade elevada, mostrando que a $T_{g}$ da fração de EVA esteja sobreposta e, portanto, não seja observada. Uma explicação da natureza bimodal desta curva pode estar relacionada com que a $T_{g}$ da mistura apareça perto da temperatura critica (ponto de turvação) e, como conseqüência disto existe separação de fases [19]. Estes resultados podem estar relacionados à formação de uma estrutura heterogênea, onde a fração de EVA (insolúvel) poderia estar na forma de micelas contendo os segmentos de PMMA estendidos e orientados na direção oposta onde se encontram as moléculas do monômero epoxídico, o que é favorecido quando a concentração de resina nas misturas é relativamente elevada ( $\leq 60 \%$, em massa). Neste caso, a separação de fases é evidente. Entretanto, o efeito de separação de fases é menos significativo como mencionado anteriormente para altas concentrações de resina ( $\geq 85 \%$, em massa). Isto foi evidente durante a preparação das misturas a temperatura ambiente. Para misturas com altas concentrações de copolímero $(>60 \%$, em massa) as moléculas de resina se encontram localizadas nos segmentos de PMMA, o que provoca a plastificação relativa do copolímero. Neste caso, a separação de fases é menos importante.

\section{CONCLUSÕES}

Foi comprovado experimentalmente que existem diferentes graus de interação entre os dois segmentos do copolímero com a resina do tipo éter diglicidílico do bisfenol-A (DGEBA). A fração de EVA do copolímero não manifestou interação aparente com a resina. No entanto, o segmento graftizado de PMMA é solúvel e apresenta fortes interações com o monômero epoxídico. A espectroscopia de relaxação dielétrica mostrou ser uma técnica muito valiosa neste sistema para o estudo das interações dos componentes das misturas estudadas neste trabalho. O sistema binário estudado apresenta grande tendência para a formação de estruturas heterogêneas que facilitam a separação de fases. 


\section{AGRADECIMENTOS}

Os autores agradecem a CNPq, FAPEMIG e FAPERJ pelo apoio financeiro recebido.

\section{BIBLIOGRAFIA}

[1] ABADYAN, M., BAGHERI, R., KOUCHAKZADEH, M.A., "Fracture toughness of a hybrid-rubbermodified epoxy I. Synergistic toughening”, Journal of Applied Polymer Science, v. 125, pp. 2467 - 2475, 2012.

[2] TRIPATHY, R., OJHA, U., FAUST, R., "Polyisobutylene modified bisphenol A diglycidyl ether based epoxy resin possessing improved mechanical properties”, Macromolecules, v. 44, pp. 6800 - 6809, 2011.

[3] KHOEE, S., HASSANI, N., “Adhesion strength improvement of epoxy resin reinforced with nanoelastomeric copolymer", Materials Science and Engineering A, v. 52, pp. 6562-6567, 2010.

[4] KUAN, H.C., DAI, J.B., MA, J., “A reactive polymer for toughening epoxy resin”, Journal of Applied Polymer Science, v. 115, 3265-3272, 2010.

[5] MAIEZ-TRIBU, S., PASCAULT, J.P., SOULÉ, E.R., et al., "Nanostructured epoxies based on the selfassembly of block copolymers: A new miscible block that can be tailored to different epoxy formulations", Macromolecules, v. 40, pp. 12681 - 273, 2007.

[6] PASCAUlT, J.P., SAUTEREAU, H., VERDU, J., et al., Thermosetting Polymers, Marcel Dekker: New York, 2002.

[7] GONZÁLEZ GARCIA, F., SOARES, B.G., WILLIAMS, R.J.J., "Poly(ethylene-co-vinyl acetate)-graftpoly(methyl methacrylate) (EVA-graft-PMMA) as a modifier of epoxy resins", Polymer International, v. 51, pp. $1340-1347,2002$.

[8] GONZÁLEZ GARCIA, F., "Modificação de polímeros epoxídicos com polímeros termoplásticos”, D.Sc., IMA-UFRJ, Rio de Janeiro, 2001.

[9] GONZÁLEZ GARCIA, F., PINTO, M.R., SOARES, B.G., "Grafting of poly(methyl methacrylate) from poly(ethylene-co-vinyl acetate) copolymer using atom transfer radical polymerization”, European Polymer Journal, v. 38, pp. $759-769,2002$.

[10] GONZÁLEZ GARCIA, F., PINTO, M.R., SOARES, B.G., et al., "Influence of aliphatic amine epoxy hardener on the adhesive properties of blends of mono-carboxylterminated poly(2-ethylhexyl acrylate-comethylmethacrylate) with epoxy resin", Journal of Applied Polymer Science, v. 117, pp. 2762 - 2770, 2010.

[11] GONZÁLEZ GARCIA, F., SOARES, B.G.; "Determination of the equivalent weight of epoxy resins based on diglycidyl ether of bisphenol A (DGEBA) by proton nuclear magnetic resonance", Polymer Testing, v. 22, pp. $51-56,2003$.

[12] SIDDHAMALLI, S.K., “Thermoset/(thermoplastic elastomer) blends: Epoxy/EVA”, Journal of Vinyl Additive Technology, v. 6, pp. 211 - 220, 2000.

[13] GÓMEZ, C.M., BUCKNALL, C.B., "Blends of poly(methyl methacrylate) with epoxy resin and an aliphatic amine hardener", Polymer, v. 34, pp. 2111 - 2117, 1993.

[14] MOUNIF, E., LIANG, G.G., WAYNE, D.C., et al., "Poly(methylmethacrylate)-modified epoxy/amine system for reactive rotational moulding: crosslinking kinetics and rheological properties", Polymer International, v. 58, pp. $954-961,2009$.

[15] WOO, E.M., WU, M.N., "Blends of diglycidylether epoxy with bisphenol - A polycarbonate or poly(methyl methacrylate). Case of miscibility with or without specific interactions", Polymer, v. 37, pp. 2485 - 2492, 1996.

[16] CAMPBELL, J.A., GOODWIN, A.A., SIMON, G.P., "Dielectric relaxation studies of miscible polycarbonate/poliester blends", Polymer, v. 42, pp. 4731 - 4741, 2001.

[17] BIZET, A., NAKAMURA, N., TERAMOTO, Y., et al., "The influence of moisture on the dielectric properties of poly(ethylene-co-vinyl alcohol)", Thermochimica Acta, v. 241, pp. 191 - 198; 1994.

[18] ZETSCHE, A., KREMER, F., JUNG, W., et al., "Dielectric study on the miscibility of binary polymer blends", Polymer, v. 31, pp. 1883 - 1887. 1990.

[19] HAYAKAMA, T., KEIICHIRO, A., "Miscibility of poly(n-butyl acrylate)/poly(propylene glycol) blends. 1. Phase behavior and dielectric relaxation", Macromolecules, v. 33, pp., 6834-6839, 2000. 